\title{
ON MIXED PROJECTIVE CURVES
}

\author{
MUTSUO OKA
}

\begin{abstract}
Let $f(\mathbf{z}, \overline{\mathbf{z}})$ be a mixed polar homogeneous polynomial of $n$ variables $\mathbf{z}=\left(z_{1}, \ldots, z_{n}\right)$. It defines a projective real algebraic variety $V:=\left\{[\mathbf{z}] \in \mathbb{C P}^{n-1} \mid f(\mathbf{z}, \overline{\mathbf{z}})=0\right\}$ in the projective space $\mathbb{C P}^{n-1}$. The behavior is different from that of the projective hypersurface. The topology is not uniquely determined by the degree of the variety even if $V$ is non-singular. We study a basic property of such a variety.
\end{abstract}

\section{INTRODUCTION}

Let $f(\mathbf{z}, \overline{\mathbf{z}})$ be a polar weighted homogeneous mixed polynomial with $\mathbf{z}=$ $\left(z_{1}, \ldots, z_{n}\right) \in \mathbb{C}^{n}$. Namely there exist integers $\left(q_{1}, \ldots, q_{n}\right)$ and $\left(p_{1}, \ldots, p_{n}\right)$ and positive integers $d_{r}, d_{p}$ such that

$$
\begin{aligned}
& f(t \circ \mathbf{z}, t \circ \overline{\mathbf{z}})=t^{d_{r}} f(\mathbf{z}, \overline{\mathbf{z}}), \quad t \circ \mathbf{z}=\left(t^{q_{1}} z_{1}, \ldots, t^{q_{n}} z_{n}\right), t \in \mathbb{R}^{+} \\
& f(\rho \circ \mathbf{z}, \overline{\rho \circ \mathbf{z}})=\rho^{d_{p}} f(\mathbf{z}, \overline{\mathbf{z}}), \quad \rho \circ \mathbf{z}=\left(\rho^{p_{1}} z_{1}, \ldots, \rho^{p_{n}} z_{n}\right), \rho \in \mathbb{C},|\rho|=1 .
\end{aligned}
$$

This gives $\mathbb{R}^{+} \times S^{1}$ action by

$$
(t, \rho) \circ \mathbf{z}=\left(t^{q_{1}} \rho^{p_{1}} z_{1}, \ldots, t^{q_{n}} \rho^{p_{n}} z_{n}\right), \quad t \rho \in \mathbb{R}^{+} \times S^{1} .
$$

The integers $d_{r}$ and $d_{p}$ are called the radial and the polar degree respectively and we denote them as $d_{r}=\operatorname{rdeg} f$ and $d_{p}=\operatorname{pdeg} f$.

We say that $f(\mathbf{z}, \overline{\mathbf{z}})$ is strongly polar weighted homogeneous if $p_{j}=q_{j}$ for $j=1, \ldots, n$. Then the associated $\mathbb{R}^{+} \times S^{1}$ action on $\mathbb{C}^{n}$ is in fact the $\mathbb{C}^{*}$ action which is defined by

$$
(\mathbf{z}, \tau)=\left(\left(z_{1}, \ldots, n\right), \tau\right) \mapsto \tau \circ \mathbf{z}=\left(z_{1} \tau^{p_{1}}, \ldots, z_{n} \tau^{p_{n}}\right), \tau \in \mathbb{C}^{*} .
$$

We say $f(\mathbf{z}, \overline{\mathbf{z}})$ is strongly polar homogeneous if further the weights satisfies the equalities $q_{j}=p_{j}=1$ for any $j$. A strongly polar weighted homogeneous polynomial $f(\mathbf{z}, \overline{\mathbf{z}})$ satisfies the equality:

$$
f((t, \rho) \circ \mathbf{z}, \overline{(t, \rho) \circ \mathbf{z}})=t^{d_{r}} \rho^{d_{p}} f(\mathbf{z}, \overline{\mathbf{z}}), \quad(t, \rho) \in \mathbb{R}^{+} \times S^{1} .
$$

Assume that $f(\mathbf{z}, \overline{\mathbf{z}})$ is a strongly polar weighted homogeneous polynomial of radial degree $d_{r}$ and of polar degree $d_{p}$ respectively and let $P=$ $\left(p_{1}, \ldots, p_{n}\right)$ be the weight vector. Let $\tilde{V}$ be the mixed affine hypersurface

$$
\tilde{V}=f^{-1}(0)=\left\{\mathbf{z} \in \mathbb{C}^{n} \mid f(\mathbf{z}, \overline{\mathbf{z}})=0\right\} .
$$

2000 Mathematics Subject Classification. 14J17, 14 N99.

Key words and phrases. Mixed weighted homogeneous, polar action, degree. 
Let $\varphi: S^{2 n-1} \backslash K \rightarrow S^{1}$ be the Milnor fibration with $K=\tilde{V} \cap S^{2 n-1}$ and let $F$ be the fiber. Recall that $\varphi(\mathbf{z})=f(\mathbf{z}, \overline{\mathbf{z}}) /|f(\mathbf{z}, \overline{\mathbf{z}})|$. Thus $F$ is defined by

$$
F=\varphi^{-1}(1)=\left\{\mathbf{z} \in S^{2 n-1} \backslash K \mid f(\mathbf{z}, \overline{\mathbf{z}})>0\right\}
$$

We can equivalently consider the global fibration $f: \mathbb{C}^{n}-\tilde{V} \rightarrow \mathbb{C}^{*}$. Then the Milnor fiber is identified with the hypersurface $f^{-1}(1)$. The monodromy map $h: F \rightarrow F$ (in either case) is defined by

$$
h(\mathbf{z})=\left(\exp \left(\frac{2 p_{1} \pi i}{d_{p}}\right) z_{1}, \ldots, \exp \left(\frac{2 p_{n} \pi i}{d_{p}}\right) z_{n}\right) .
$$

We consider also the wighted projective hypersurface $V$ defined by

$$
V=\left\{\left(z_{1}: z_{2}: \cdots: z_{n}\right) \in \mathbb{C P}(P)^{n-1} \mid f(\mathbf{z}, \overline{\mathbf{z}})=0\right\}
$$

where $\mathbb{C P}(P)^{n-1}$ is the weighted projective space defined by the equivalence induced by the above $\mathbb{C}^{*}$ action:

$$
\mathbf{z} \sim \mathbf{w} \Longleftrightarrow \exists \tau \in \mathbb{C}^{*}, \mathbf{w}=\tau \circ \mathbf{z} .
$$

It is well-known that $\mathbb{C P}(P)^{n-1}$ is an orbifold with at most cyclic quotient singularities.

By (11), $\mathbf{z} \in f^{-1}(0)$ and $\mathbf{z}^{\prime} \sim \mathbf{z}$, then $\mathbf{z}^{\prime} \in f^{-1}(0)$. Thus the hypersurface $V=\left\{[\mathbf{z}] \in \mathbb{C P}^{n-1}(P) \mid f(\mathbf{z})=0\right\}$ is well-defined. Consider the quotient map $\pi: S^{2 n-1} \rightarrow \mathbb{C P}(P)^{n-1}$ or $\pi: \mathbb{C}^{n} \backslash\{O\} \rightarrow \mathbb{C P}(P)^{n-1}$. For the brevity's sake, we denote the restrictions $\pi \mid F: F \rightarrow \mathbb{C P}^{n-1} \backslash V$ and $\pi \mid K: K \rightarrow V$ by the same $\pi$. We are interested in the topology of $V$ and the relation with the Milnor fibration.

In this paper, we consider only the case of strictly polar homogeneous polynomials. It turns out that the topology of the smooth projective mixed hypersurface $V$ is not an invariant of the degree $d_{r}, d_{p}$. However we will show that the degree of $V$ is equal to the polar degree $d_{p}$ (Theorem 11).

In $\S 5$, we study the case $n=3$. In this case, let $g$ be the genus of the mixed curve $V$ and put $q=d_{p}$. Then it is known that the following inequality holds (known as the Thom's conjecture and proved by Kronheimer-Mrowka, 4]).

$$
g \geq \frac{(q-1)(q-2)}{2} .
$$

We also give examples of mixed projective curves in $\mathbb{C P}^{2}$ which shows that $g$ is unbounded when $q$ is fixed.

This is a continuation of our papers [8, 9] and we use the same notations.

\section{Milnor fibration And the Hopf fibration}

2.1. Canonical orientation. It is well known that a complex analytic smooth variety has a canonical orientation which comes from the complex structure (see for example p.18, [3]). Let $\tilde{V}=f^{-1}(0)$ be a mixed hypersurface. Take a point $\mathbf{a} \in \tilde{V}$. We say that $\mathbf{a}$ is a mixed singular point of $\tilde{V}$, if a is a critical point of the mapping $f: \mathbb{C}^{n} \rightarrow \mathbb{C}$. Otherwise, a is a mixed regular point. Note that a point $\mathbf{a} \in \tilde{V}$ to be a regular point as a 
point of a real analytic variety is a necessary condition but not a sufficient condition for the regularity as a point on a mixed variety. Recall that $\mathbf{a}$ is a mixed singular point if and only if $d f_{\mathbf{a}}: T_{\mathbf{a}} \mathbb{C}^{n} \rightarrow T_{f(\mathbf{a})} \mathbb{C}$ is surjective. This is equivalent to the existence of a complex number $\alpha \in \mathbb{C}$ with $|\alpha|=1$ such that

$$
\overline{d f}(\mathbf{a}, \overline{\mathbf{a}})=\alpha \bar{d} f(\mathbf{a}, \overline{\mathbf{a}}) \quad \text { i.e., } \quad \frac{\overline{\partial f}}{\partial z_{j}}(\mathbf{a}, \overline{\mathbf{a}})=\alpha \frac{\partial f}{\partial \bar{z}_{j}}(\mathbf{a}, \overline{\mathbf{a}}), j=1, \ldots, n
$$

([7]). We assert that

Proposition 1. There is a canonical orientation on the smooth part of a mixed hypersurface.

Proof. Take a regular point $\mathbf{a} \in \tilde{V}$. The normal bundle $\mathcal{N}$ of $\tilde{V} \subset \mathbb{C}^{n}$ has a canonical orientation so that $d f_{\mathbf{a}}: \mathcal{N}_{\mathbf{a}} \rightarrow T_{f(\mathbf{a}, \overline{\mathbf{a}})} \mathbb{C}$ is an orientation preserving isomorphism. This gives a canonical orientation on $\tilde{V}$ so that the ordered union of the oriented frames $\left\{v_{1}, \ldots, v_{2 n-2}, n_{1}, n_{2}\right\}$ of $T_{\mathbf{a}} \mathbb{C}^{n}$ is the orientation of $\mathbb{C}^{n}$ if and only if $\left\{v_{1}, \ldots, v_{2 n-2}\right\}$ is an oriented frame of $T_{\mathbf{a}} \tilde{V}$ where $\left\{n_{1}, n_{2}\right\}$ is an oriented frame of normal vectors.

Consider a mixed homogeneous hypersurface $\tilde{V}$ and let $V$ be the corresponding mixed projective hypersurface.

Proposition 2. Let $\mathbf{a} \in \tilde{V} \backslash\{O\}$. Then $\mathbf{a} \in \tilde{V}$ is a mixed singular point of $\tilde{V}$ if and only if $\pi(\mathbf{a}) \in V$ is a mixed singular point.

Proof. Assume that $\mathbf{a}=\left(a_{1}, \ldots, a_{n}\right) \in \widetilde{V}$ and $a_{n} \neq 0$ for simplicity. Let $u_{j}=z_{j} / z_{n}, 1 \leq j \leq n-1$ be the affine coordinates of the chart $U_{n}=\left\{z_{n} \neq\right.$ $0\}$. Then $V \cap U_{n}$ is defined by

$$
V \cap U_{n}=\left\{\mathbf{u} \in \mathbb{C}^{n-1} \mid g(\mathbf{u}, \overline{\mathbf{u}})=0\right\}
$$

where $\mathbf{u}=\left(u_{1}, \ldots, u_{n-1}\right)$ and $g(\mathbf{u}, \overline{\mathbf{u}})$ is defined by

$$
g(\mathbf{u}, \overline{\mathbf{u}}):=f\left(\mathbf{u}^{\prime}, \overline{\mathbf{u}^{\prime}}\right), \quad \mathbf{u}^{\prime}:=(\mathbf{u}, 1) .
$$

Putting $q+2 r=\operatorname{rdeg} f$ and $q=\operatorname{pdeg} f$, we observe that

$$
g(\mathbf{u}, \overline{\mathbf{u}})=f(\mathbf{z}, \overline{\mathbf{z}}) /\left(z_{n}^{q+r} \bar{z}_{n}^{r}\right) .
$$

Here $d_{r}-d_{p}=2 r$ and $d_{p}=q$. Write $a_{n}=r_{n} \exp \left(\theta_{n} i\right)$ in the polar coordinate. Consider the hyperplane section $\widetilde{U}_{n}=\mathbb{C}^{n} \cap\left\{z_{n}=a_{n}\right\}$ and $\widetilde{f}:=\left.f\right|_{\widetilde{U}_{n}}$. Then we have the commutative diagram:

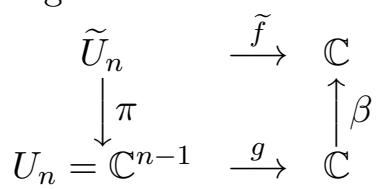

where $\beta$ is the multiplication with $r_{n}^{d_{r}} \exp \left(i d_{p} \theta_{n}\right)$. This follows from the equality (2). Put $\alpha:=\pi(a) \in U_{n} \cap V$. Then the above diagram says that $d \widetilde{f}_{a}: T_{a} \widetilde{U}_{n} \rightarrow T_{O} \mathbb{C}$ is surjective if and only if $d g_{\alpha}: T_{\alpha} U_{n} \rightarrow T_{O} \mathbb{C}$ 
is surjective. On the other hand, $T_{a} \mathbb{C}^{n}$ is a direct sum of $T_{a} \widetilde{U}_{n}$ and the tangent space of the $\mathbb{R}^{+} \times S^{1}$ orbit at $a$ and the latter space is in the kernel of $d f_{a}: T_{a} \mathbb{C}^{n} \rightarrow T_{O} \mathbb{C}$, as $\widetilde{V}$ is invariant by the $\mathbb{R}^{+} \times S^{1}$-action. This shows that the surjectivities of two tangential maps $d f_{a}: T_{a} \mathbb{C}^{n} \rightarrow T_{O} \mathbb{C}$ and $d g_{\alpha} U_{n} \rightarrow T_{O} \mathbb{C}$ are equivalent. Thus $a \in \widetilde{V}$ is mixed non-singular if and only if $\alpha \in V$ is mixed nonsingular.

Now we consider the canonical orientation of $V$. First we recall that the orientation of $\mathbb{C}^{*}$ is given by the frame $\left\{\frac{\partial}{\partial r}, \frac{\partial}{\partial \theta}\right\}$ where $(r, \theta)$ is the polar coordinates of $\mathbb{C}^{*}$. The orientation of $\mathbb{C P}^{n-1}$ as a complex manifold and the orientation of $\mathbb{C P}^{n-1}$ coming from the Hopf bundle using the local bundle structure $U_{j} \times \mathbb{C}^{*}$ is the same. Using the orientation of the affine hypersurface $\widetilde{V}$ and the local product structure of the restriction of the Hopf bundle over $V$, we have a canonical orientation on (the smooth part of ) $V$. One can easily see that the orientation as the local mixed hypersurface $V \cap U_{n}=$ $g^{-1}(0) \subset U_{n}$ is the same with the above orientation.

2.2. Milnor fiber. Consider the Hopf fibration $\pi: S^{2 n-1} \rightarrow \mathbb{C P}^{n-1}$ and its restriction to the Milnor fiber $F=\left\{\mathbf{z} \in S^{2 n-1} \mid f(\mathbf{z}, \overline{\mathbf{z}})>0\right\}$. As $f$ is polar weighted, it is easy to see that $\pi: F \rightarrow \mathbb{C P}^{n-1} \backslash V$ is a cyclic covering of order $d_{p}$ and the covering transformation is generated by the monodromy map

Thus we have

$$
h: F \rightarrow F, \quad \mathbf{z} \mapsto \exp \left(\frac{2 \pi i}{d_{p}}\right) \cdot \mathbf{z} .
$$

Proposition 3. (1) $\chi(F)=d_{p} \chi\left(\mathbb{C P}^{n-1} \backslash V\right)$.

(2) $\chi\left(\mathbb{C P}^{n-1} \backslash V\right)=n-\chi(V)$ and $\chi(V)=n-\chi(F) / d_{p}$.

(3) We have the following exact sequence.

$$
1 \rightarrow \pi_{1}(F) \stackrel{\pi_{\sharp}}{\longrightarrow} \pi_{1}\left(\mathbb{C P}^{n-1} \backslash V\right) \rightarrow \mathbb{Z} / d_{p} \mathbb{Z} \rightarrow 1 .
$$

Corollary 4. If $d_{p}=1$, the projection $\pi: F \rightarrow \mathbb{C P}^{n-1} \backslash V$ is a diffeomorphism.

The monodromy map $h: F \rightarrow F$ gives free $\mathbb{Z} / d_{p} \mathbb{Z}$ action on $F$. Thus using the periodic monodromy argument in [5], we get

Proposition 5. The zeta function of $h: F \rightarrow F$ is given by

$$
\zeta(t)=\left(1-t^{d_{p}}\right)^{-\chi(F) / d_{p}}
$$

In particular, if $d_{p}=1, h=\mathrm{id}_{\mathrm{F}}$ and $\zeta(t)=(1-t)^{-\chi(F)}$.

\section{TOPOlOGY OF MIXED PROJECTIVE HYPERSURFACE}

We are interested in the topology of the mixed projective hypersurface. Assume that $f(\mathbf{z}, \overline{\mathbf{z}})$ is a mixed homogeneous polynomial of radial degree $d_{r}$ and of polar degree $q$. Let $V$ be the corresponding projective hypersurface $V=\{f(\mathbf{z}, \overline{\mathbf{z}})=0\} \subset \mathbb{C P}^{n-1}$. In the case of smooth complex algebraic 
hypersurfaces, the topology of $F$ or $V$ are determined by the degree $q$. In the case of mixed hypersurfaces, we will see later that the degree $q$ do not determine the topology of the Milnor fibering of $f$ or the topology of $V$.

3.1. Isolated singularity case. We consider a mixed strongly polar homogeneous polynomial $f(\mathbf{z}, \overline{\mathbf{z}})$ of polar degree $q$ and we assume that $\widetilde{V}=f^{-1}(0)$ has an isolated mixed singularity at the origin.

Assume that $F=f^{-1}(1)$ has a homotopy type of a bouquet of spheres of dimension $n-1$.

Proposition 6. Under the above assumption, the projective mixed hypersurface has the following homology groups:

$$
H_{j}(V)= \begin{cases}\mathbb{Z}, & j: \text { even }, j \leq 2(n-2), j \neq n-2 \\ 0, & j: \text { odd }, j<2(n-2), j \neq n-2 .\end{cases}
$$

The middle homology group $H_{n-2}(V)$ is determined by $\chi(F)$ up to the torsion part.

Proof. First, by the assumption, $\tilde{H}_{j}(F)=0$ for $j \leq n-2$ and $H_{n-1}(F)=\mathbb{Z}^{\mu}$ where

$$
\mu=(-1)^{n-1}(\chi(F)-1)
$$

By the Wang sequence of the Milnor fibration

$$
\cdots \rightarrow H_{j+1}\left(S^{2 n-1} \backslash K\right) \rightarrow H_{j}(F) \stackrel{h_{*}-\mathrm{id}}{\longrightarrow} H_{j}(F) \rightarrow H_{j}\left(S^{2 n-1} \backslash K\right) \rightarrow \ldots
$$

we see that

$$
H_{j}\left(S^{2 n-1} \backslash K\right)=0, \quad j<n-2
$$

As for $H_{n-2}\left(S^{2 n-1} \backslash K\right)$, it is isomorphic to the cokernel of $h_{*}-$ id : $\mathrm{H}_{\mathrm{n}-1}(\mathrm{~F}) \rightarrow \mathrm{H}_{\mathrm{n}-1}(\mathrm{~F})$ and the rank of this cokernel can be computed from the zeta function and the characteristic polynomial $P_{n-1}(t)$ which are related by

$$
\left(t^{d_{p}}-1\right)^{\chi(F) / d_{p}}=\zeta(t)=\frac{P_{n-1}(t)^{(-1)^{n-2}}}{(t-1)} .
$$

We leave the calculation to the reader. By the Alexander duality, we get

$$
\tilde{H}_{j}(K)=0, \quad j<n-2 .
$$

Now the assertion follows from the Gysin sequence of the Hopf fibration $\pi: K \rightarrow V:$

$$
\cdots \rightarrow H_{j}(K) \rightarrow H_{j}(V) \rightarrow H_{j-2}(V) \rightarrow H_{j-1}(K) \rightarrow \ldots
$$

The argument is exactly same as that for a projective hypersurface (see [6])

Remark 7. Assume that a mixed function $f(\mathbf{z}, \overline{\mathbf{z}})$ is strongly non-degenerate. It is an open problem if (i) $F$ is $(n-2)$-connected, and (ii) $F$ has a homotopy type of $C W$-complex of dimension $n-1$. 
3.2. Solutions and points in $\mathbb{C P}^{1}$. Let us consider the case $n=2$. Let $\mathcal{M}(q+2 r, q ; 2)$ be the set of the mixed polar homogeneous polynomials with the radial degree $q+2 r$ and the polar degree $q$. Let $f(\mathbf{z}, \overline{\mathbf{z}})$ be a nondegenerate strongly polar homogeneous polynomial in $\mathcal{M}(q+2 r, q ; 2)$ where $\mathbf{z}=\left(z_{1}, z_{2}\right)$. For brevity, we assume that $q, r>0$. We are interested to compute the number of points $V:=\{f(\mathbf{z}, \overline{\mathbf{z}})=0\}$. This number is equal to the number of link components of $S^{3} \cap \widetilde{V}$ where $\tilde{V}=f^{-1}(0) \subset \mathbb{C}^{2}$ and we denoted this number by $\operatorname{lkn}(\widetilde{V})$ in $[8$. In general, $f$ takes the following form:

$$
f(\mathbf{z}, \overline{\mathbf{z}})=\sum_{\nu, \mu} c_{\nu, \mu} z_{1}^{\nu_{1}} z_{2}^{\nu_{2}} \bar{z}_{1}^{\mu_{1}} \bar{z}_{2}^{\mu_{2}}
$$

where $\nu_{1}+\nu_{2}=\mu_{1}+\mu_{2}+q$ and $\mu_{1}+\mu_{2}=r$. We may assume that there are no points of $V$ with $z_{2}=0$. Thus we may consider the coordinate chart $\left\{z_{2} \neq 0\right\}$ with $z=z_{1} / z_{2}$ as the coordinate. To know the exact number of points of $V$, we need to know the number of complex solutions of the mixed polynomial:

$$
\left\{z \in \mathbb{C} \mid \sum_{\nu_{1}, \mu_{1}} c_{\nu_{1}, \mu_{1}}^{\prime} z^{\nu_{1}} \bar{z}^{\mu_{1}}=0\right\}, \quad \nu_{1}+\mu_{1} \leq q+2 r, \mu_{1} \leq r
$$

where $c_{\nu_{1}, \mu_{1}}^{\prime}=\sum_{\nu_{2}, \mu_{2}} c_{\nu, \mu}$. In fact, the number of solutions is not so easy to be computed as in the case of complex polynomials.

Example 8. Consider the equation:

$$
-2 z^{2} \bar{z}+t z^{2}+1=0, t \in \mathbb{C} .
$$

This example is considered in Example 59 of our previous paper [8]. We can see that for a "small" $t$, we have only one solution. For example $t=0$, $z=\frac{1}{\sqrt[3]{2}}$. For a "large" $t$, we have three solutions. (For real numbers, $t$ is "small" if $-3<t<1$.) For example, put $t=3$. Then we get $z=a$ and $1 / 9 \pm \sqrt{26} i / 9$ where $a$ is the real root of $-2 a^{3}+3 a^{2}+1=0$.

This example tells us that the number of solutions depends on the coefficients. However we have the following observation.

Proposition 9. Assume that $f(\mathbf{z}, \overline{\mathbf{z}}) \in \mathcal{M}(q+2 r, q ; 2)$ and let $V=\{[\mathbf{z}] \in$ $\left.\mathbb{C P}^{1} \mid f(\mathbf{z}, \overline{\mathbf{z}})=0\right\}$ and let $F=f^{-1}(1) \subset \mathbb{C}^{2}$. Then $\alpha:=\sharp V$ can take (at least) $q, q+2, \ldots, q+2 r$. The corresponding Euler characteristic of $F$ are $\chi(F)=q(2-\alpha)$.

Proof. We consider the basic two strongly polar homogeneous polynomials:

$$
\begin{aligned}
& f_{q, j}:=z_{1}^{q+j} \bar{z}_{1}^{j}+z_{2}^{q+j} \bar{z}_{2}^{j} \in \mathcal{M}(q+2 j, q ; 2) \\
& k_{\ell}:=\left(z_{1}^{\ell}-\beta z_{2}^{\ell}\right)\left(\bar{z}_{1}^{\ell}-\gamma \bar{z}_{2}^{\ell}\right) \in \mathcal{M}(2 \ell, 0 ; 2), \beta, \gamma \in \mathbb{C}^{*}
\end{aligned}
$$

By Theorem 10, [7], $f_{q, j}$ is strongly polar homogeneous and $\operatorname{lkn}\left(\widetilde{V}\left(f_{q, j}\right)\right)=q$. $k_{\ell}$ is obviously strongly polar homogeneous of degree 0 and $\operatorname{lkn}\left(\widetilde{\left.\left(k_{\ell}\right)\right)}=2 \ell\right.$. Thus $f_{q, j} k_{r-j}$ for $0 \leq j \leq r$ is strongly polar homogeneous polynomial in 
$\mathcal{M}(q+2 r, q ; 2)$ and non-degenerate as long as $V\left(f_{q, j}\right) \cap V\left(k_{\ell}\right)=\emptyset$. As $2 \ell+(q+2 j)=q+2 r, \operatorname{lkn}\left(\widetilde{V}\left(f_{q, j} k_{r-j}\right)\right)=q+2(r-j)$ with $j=1, \ldots, r$. The latter assertion follows from Lemma 64 of 8 .

Conjecture 10. The possible $\alpha$ for $f \in \mathcal{M}(q+2 r, q ; 2)$ is exactly $\{q, q+$ $2, \ldots, q+2 r\}$.

\section{Degree of MIXED PROJECTIVE hypersurfaceS}

Suppose that $f(\mathbf{z}, \overline{\mathbf{z}}) \in \mathcal{M}(q+2 r, q ; n)$ be a strongly polar homogeneous polynomial and let

$$
V=\left\{\mathbf{z} \in \mathbb{C P}^{n-1} \mid f(\mathbf{z}, \overline{\mathbf{z}})=0\right\} .
$$

We assume that the singular locus $\Sigma V$ of $V$ is either empty or $\operatorname{codim}_{\mathbb{R}} \Sigma \mathrm{V} \geq$ 2. We have observed that $V \backslash \Sigma V \subset \mathbb{C P}^{n-1}$ is canonically oriented so that the union of the oriented frame of $T_{P} V$, say $\left\{v_{1}, \ldots, v_{2(n-2)}\right\}$ and the frame of normal bundle $\left\{w_{1}, w_{2}\right\}$ which is compatible with the local defining complex function $g_{j}$ on the affine chart $U_{j}=\left\{z_{j} \neq 0\right\}$ is the oriented frame of $\mathbb{C P}^{n-1}$. (Recall that $g_{j}$ is a mixed function of the variables $u_{i}=z_{i} / z_{j}, i \neq j$ defined by $g_{j}(\mathbf{u}, \overline{\mathbf{u}})=f(\mathbf{z}, \overline{\mathbf{z}}) / z_{j}^{q+r} / \bar{z}_{j}^{r}$.) Thus it has a fundamental class $[V] \in H_{2 n-4}(V ; \mathbb{Z})$ by Borel-Haefliger [1]. The topological degree of $V$ is the integer $d$ so that $\iota_{*}[V]=d\left[\mathbb{C P}^{n-2}\right]$ where $\iota: V \rightarrow \mathbb{C P}^{n-1}$ is the inclusion map and $\left[\mathbb{C P}^{n-2}\right]$ is the homology class of a canonical hyperplane $\mathbb{C P}^{n-2}$.

The main result of this paper is:

Theorem 11. The topological degree of $V$ is equal to the polar degree $q$. Namely the fundamental class $[V]$ corresponds to $q\left[\mathbb{C P}^{n-2}\right] \in H_{2(n-2)}\left(\mathbb{C P}^{n-1}\right)$ by the inclusion mapping $\iota_{*}$.

Proof. Suppose that $f$ is a non-degenerate mixed polynomial in $\mathcal{M}(q+$ $2 r, q ; n)$. Take a generic 1-dimensional complex line $L$ which is isomorphic to $\mathbb{C P}^{1}$. Then the degree is given by the intersection number $[V] \cdot[L]$. Now, changing the coordinates if necessary, we may assume that

$$
L: z_{j}=a_{j 1} z_{1}+a_{j 2} z_{2}, j=3, \ldots, n .
$$

Substituting (3) in $f(\mathbf{z}, \overline{\mathbf{z}})$ to eliminate the variables $z_{3}, \ldots, z_{n}$, we see that the intersection $V \cap L$ are described by

$$
g\left(z_{1}, z_{2}, \bar{z}_{1}, \bar{z}_{2}\right)=0, \quad\left[z_{1}: z_{2}\right] \in L=\mathbb{C P}^{1} .
$$

As $g$ is still polar homogeneous in $z_{1}, z_{2}$ under the restriction to $L, g$ is written as

$$
g(\mathbf{w}, \overline{\mathbf{w}})=\left.f(\mathbf{w}, \overline{\mathbf{w}})\right|_{L}=\sum_{\nu, \mu} c_{\nu, \mu} z_{1}^{\mu_{1}} z_{2}^{\nu_{2}} \bar{z}_{1}^{\mu_{1}} \bar{z}_{2}^{\mu_{2}}, \mathbf{w}=\left(z_{1}, z_{2}\right),
$$

where the summation are for the multi-integers $\nu=\left(\nu_{1}, \nu_{2}\right), \mu=\left(\mu_{1}, \mu_{2}\right)$ such that

$$
|\nu|+|\mu|=q+2 r,|\nu|-|\mu|=q,|\nu|=\nu_{1}+\nu_{2},|\mu|=\mu_{1}+\mu_{2} .
$$


Thus the polynomial $g\left(z_{1}, z_{2}, \bar{z}_{1}, \bar{z}_{2}\right)$ is a polar homogeneous polynomial of polar degree $q$. Taking a linear change of coordinates if necessary, we may assume that the intersections are in the affine space $z_{2} \neq 0$. This implies that $g$ has a monomial $z_{1}^{q+r} \bar{z}_{1}^{r}$ with a non-zero coefficient. Use the affine coordinate $w=z_{1} / z_{2}$ for the affine coordinate $\left\{z_{2} \neq 0\right\}$. Then $g$ takes the form:

$$
g(w, \bar{w})=c_{0} w^{q+r}+c_{1} w^{q+r-1}+\cdots+c_{q+r}
$$

where $c_{j}$ is a polynomial in $\bar{w}$ such that $\operatorname{deg}_{\bar{w}} c_{j} \leq r$ and by the assumption, we have that $c_{0}=\sum_{i=0}^{r} c_{0 i} \bar{w}^{i}$ with $c_{0 r} \neq 0$. Let $\left\{\alpha_{1}, \ldots, \alpha_{m}\right\}=$ $\{w \mid g(\alpha, \bar{\alpha})=0\}$. We can see easily that

$$
I\left(V, L ; \alpha_{j}\right)=\frac{1}{2 \pi} \int_{\left|w-\alpha_{j}\right|=\varepsilon} \operatorname{Gauss}(g) d \theta
$$

where $w-\alpha_{j}=\varepsilon \exp (i \theta)$ and $\operatorname{Gauss}(g)(w, \bar{w})=\theta^{\prime}$ with $\theta^{\prime}=\arg (g(w, \bar{w}))$ and $\varepsilon$ is a sufficiently small positive number. In fact, the orientation of $V$ is defined so that a frame $\left\{v_{1}, \ldots, v_{2 n-4}\right\}$ at $\alpha_{j}$ is positive if and only if $\left\{v_{1}, \ldots, v_{2 n-4}, n_{1}, n_{2}\right\}$ are positive where $n_{1}, n_{2}$ are frames of the normal bundle of $V$ oriented by $f$. On the other hand, $\left\{\frac{\partial}{\partial x}, \frac{\partial}{\partial y}\right\}$ is also a frame of the normal bundle where $w=x+i y$. The orientation $\left\{n_{1}, n_{2}\right\}$ and $\left\{\frac{\partial}{\partial x}, \frac{\partial}{\partial y}\right\}$ are compatible if and only if the Gauss map at $\alpha_{j}$ has the positive rotation.

Topologically the intersection number is the mapping degree of the Gauss mapping, considered as:

$$
\operatorname{Gauss}(g):\left\{\left|w-\alpha_{j}\right|=\varepsilon\right\} \cong S^{1} \rightarrow S^{1} .
$$

Take a sufficiently large positive number $R$. Then by a standard argument, we see that

$$
\sum_{j=1}^{m} \frac{1}{2 \pi} \int_{\left|w-\alpha_{j}\right|=\varepsilon} \operatorname{Gauss}(g) d \theta=\frac{1}{2 \pi} \int_{|w|=R} \operatorname{Gauss}(g) d \theta .
$$

The right hand side is equal to the mapping degree of

$$
\operatorname{Gauss}(\mathrm{g}):\{|w|=R\} \cong S^{1} \rightarrow S^{1}
$$

which is equal to $q$ by the next Lemma which completes the proof.

4.1. Residue formula for a monic mixed polynomial. Let $g(w, \bar{w})=$ $\sum_{a, b} c_{a, b} w^{a} \bar{w}^{b}$ be a mixed polynomial. Put $d=\max \left\{a+b \mid c_{a, b} \neq 0\right\}$ and we call $d$ the radial degree of $g$. We say that $g$ is a monic mixed polynomial of degree $d$ if $g$ has a unique monomial of radial degree $d$.

Lemma 12. Assume that $g(w, \bar{w})$ is a monic mixed polynomial of degree $d$ which is written as

$$
\begin{gathered}
g(w, \bar{w})=c_{0}(\bar{w}) w^{q+r}+c_{1}(\bar{w}) w^{q+r-1}+\cdots+c_{q+r} \\
c_{j}(\bar{w}) \in \mathbb{C}[\bar{w}], \operatorname{rdeg}_{\bar{w}} c_{j} \leq r, j=0, \ldots, q+r \\
c_{0}(\bar{w})=c_{0 r} \bar{w}^{r}+\cdots+c_{00}, c_{0 r} \neq 0
\end{gathered}
$$


with $d=q+2 r$. Then

$$
\frac{1}{2 \pi} \int_{|w|=R} \operatorname{Gauss}(g) d \theta=q .
$$

Proof. Consider the family $g_{t}(w, \bar{w})=(1-t) g(w, \bar{w})+t h(w, \bar{w})$ with $h(w, \bar{w}):=$ $c_{0 r} w^{q+r} \bar{w}^{r}$. For a sufficiently large $R$, this gives a homotopy of the two Gauss maps of $\left.g\right|_{|w|=R}$ and $\left.h(w, \bar{w})\right|_{|w|=R}$. The rotation number of the Gauss map $\left.h(w, \bar{w})\right|_{|w|=R}$ is obviously $q$. This proves the assertion.

\section{Mixed PRojective CURVES}

In this section, we study basic examples in the projective surface $\mathbb{C P}^{2}$. Thus we assume that $n=3$.

5.1. Milnor fibers. Let $f(\mathbf{z}, \overline{\mathbf{z}})$ be a strongly polar weighted homogeneous polynomial in three variables $\mathbf{z}=\left(z_{1}, z_{2}, z_{3}\right)$. Let $F=f^{-1}(1) \subset \mathbb{C}^{3}$ be the Milnor fiber.

Proposition 13. Assume that $f$ is 1-convenient (see [8] for the definition) non-degenerate, polar weighted homogeneous polynomial with isolated mixed singularities at the origin and we assume that $f$ is either of a join type or of a simplicial type which are described below.

Join type : $f_{1}(\mathbf{z}, \overline{\mathbf{z}})=g\left(z_{1}, z_{2}, \bar{z}_{1}, \bar{z}_{2}\right)+z_{3}^{a_{3}+b_{3}} \bar{z}_{3}^{b_{3}}$,

$$
\text { Simplicial type: }\left\{\begin{array}{l}
f_{2}(\mathbf{z}, \overline{\mathbf{z}})=z_{1}^{a_{1}+b_{1}} \bar{z}_{1}^{b_{1}} z_{2}+z_{2}^{a_{2}+b_{2}} \bar{z}_{2}^{b_{2}} z_{3}+z_{3}^{a_{3}+b_{3}} \bar{z}_{3}^{b_{3}} \\
f_{2}^{\prime}(\mathbf{z}, \overline{\mathbf{z}})=z_{1}^{a_{1}+b_{1}} \bar{z}_{1}^{b_{1}} \bar{z}_{2}+z_{2}^{a_{2}+b_{2}} \bar{z}_{2}^{b_{2}} \bar{z}_{3}+z_{3}^{a_{3}+b_{3}} \bar{z}_{3}^{b_{3}} \\
f_{3}(\mathbf{z}, \overline{\mathbf{z}})=z_{1}^{a_{1}+b_{1}} \bar{z}_{1}^{b_{1}} z_{2}+z_{2}^{a_{2}+b_{2}} \bar{z}_{2}^{b_{2}} z_{3}+z_{3}^{a_{3}+b_{3}} \bar{z}_{3}^{b_{3}} z_{1} \\
f_{3}^{\prime}(\mathbf{z}, \overline{\mathbf{z}})=z_{1}^{a_{1}+b_{1}} \bar{z}_{1}^{b_{1}} \bar{z}_{2}+z_{2}^{a_{2}+b_{2}} \bar{z}_{2}^{b_{2}} \bar{z}_{3}+z_{3}^{a_{3}+b_{3}} \bar{z}_{3}^{b_{3}} \bar{z}_{1}
\end{array}\right.
$$

with $a_{i}, b_{i}>0, i=1,2,3$ where $g\left(z_{1}, z_{2}, \bar{z}_{1}, \bar{z}_{2}\right)$ is a non-degenerate polar weighted homogeneous polynomial. Then the Milnor fibers $F\left(f_{i}\right), i=$ $1, \ldots, 3$ and $F\left(f_{i}^{\prime}\right), i=2,3$ are simply connected and they have homotopy types of bouquets of spheres $S^{2} \vee \cdots \vee S^{2}$. Let $\mu_{g}$ be the Milnor number of $g$. The Euler characteristics and the Milnor numbers are given as follows.

$$
\begin{aligned}
& \chi\left(F\left(f_{1}\right)\right)=\left(a_{3}-1\right) \mu_{g}+1, \quad \mu\left(f_{1}\right)=\left(a_{3}-1\right) \mu_{g} \\
& \chi\left(F\left(f_{2}\right)\right)=\chi\left(F\left(f_{2}^{\prime}\right)\right)=a_{1} a_{2} a_{3}-a_{2} a_{3}+a_{3}, \\
& \mu\left(f_{2}\right)=\mu\left(f_{2}^{\prime}\right)=\chi\left(F\left(f_{2}\right)\right)-1, \\
& \chi\left(F\left(f_{3}\right)\right)=a_{1} a_{2} a_{3}+1, \quad \mu\left(f_{3}\right)=a_{1} a_{2} a_{3}, \\
& \chi\left(F\left(f_{3}^{\prime}\right)\right)=a_{1} a_{2} a_{3}-1 \quad \mu\left(f_{3}^{\prime}\right)=a_{1} a_{2} a_{3}-2,
\end{aligned}
$$

Proof. We consider first $F_{1}=f_{1}^{-1}(1)$ where

$$
f_{1}(\mathbf{z}, \overline{\mathbf{z}})=g\left(z_{1}, z_{2}, \bar{z}_{1}, \bar{z}_{2}\right)+z_{3}^{a_{3}+b_{3}} \bar{z}_{3}^{b_{3}}
$$

where $g\left(z_{1}, z_{2}, \bar{z}_{1}, \bar{z}_{2}\right)$ is a convenient non-degenerate polar weighted homogeneous polynomial. For two variables case, the Milnor fiber $F_{g}$ of $g\left(z_{1}, z_{2}\right)$ has the homotopy type of a bouquet of $S^{1}$ as it is a connected open Riemann 
surface (Proposition 38, 8 ). Let $\mu_{g}$ be the Milnor number (=the first Betti number) of $F_{g}$. Then the Milnor fiber $F_{1}$ of $f_{1}$ is homotopic to the join $F_{g} * \Omega_{a}$ where $\Omega_{a}$ is the set of $a$-th roots of unity ([2]). This join is obviously homotopic to a bouquet of $\mu_{g}(a-1) S^{2}$ spheres.

Consider $F_{2}=f_{2}^{-1}(1)$ or $F_{2}^{\prime}=f_{2}^{-1}(1)$. The Euler characteristic can be easily computed from the additivity of the Euler characteristics, applied on the toric stratification

$$
F_{2}=F_{2}^{*\{1,2,3\}} \amalg F_{2}^{*\{2,3\}} \amalg F_{2}^{*\{3\}}, \quad F_{2}^{\prime}=F_{2}^{*\{1,2,3\}} \amalg F_{2}^{*\{2,3\}} \amalg F_{2}^{*\{3\}},
$$

and Theorem 10 of [7], where $F_{2}^{* I}$ is defined by $F_{2} \cap \mathbb{C}^{* I}$ and

$$
\mathbb{C}^{* I}=\left\{\mathbf{z} \in \mathbb{C}^{3} \mid z_{i} \neq 0, i \in I, z_{j}=0, j \notin I\right\}
$$

for $I \subset\{1,2,3\}$.

The Euler characteristics of $F_{3}=f_{3}^{-1}(1)$ and $F_{3}^{\prime}=f_{3}^{\prime-1}(1)$ can be computed in the exact same way. The assertion on the homotopy types are now obtained simultaneously as follows. First $F_{j}^{*\{1,2,3\}}$ and $F_{j}^{\prime *\{1,2,3\}}$ are CW-complex of the dimension 2 by Theorem 10, [7]. The secondly $F_{j}$ and $F_{j}^{\prime}$ are simply connected by the 1-convenience assumption ([7]). Using the above decomposition and Mayer-Vietoris exact sequences, we see that the (reduced) homology groups are non-trivial only on the dimension 2 and no torsion on $H_{2}\left(F_{j}\right)$ and $H_{2}\left(F_{j}^{\prime}\right)$ for $j=2,3$. Thus by the Whitehead theorem (see for example [10]), we conclude that $F_{j}$ and $F_{j}^{\prime}$ are homotopic to bouquets of two dimensional spheres.

5.2. Projective mixed curves. We consider projective curves of degree $q$ :

$$
C=\left\{\left[z_{1}: z_{2}: z_{3}\right] \in \mathbb{C P}^{2} \mid f\left(z_{1}, z_{2}, z_{3}\right)=0\right\}
$$

where $f$ is a strongly polar homogeneous polynomial with $\operatorname{pdeg} f=q$. We have seen that the topological degree of $C$ is $q$ by Theorem 11. The genus $g$ of $C$ is not an invariant of $q$. Recall that for a differentiable curve $C$ of genus $g$, embedded in $\mathbb{C P}^{2}$, with the topological degree $q$, we have the following Thom's inequality, which was conjectured by Thom and proved by for example Kronheimer-Mrowka [4]:

$$
g \geq \frac{(q-1)(q-2)}{2}
$$

where the right side number is the genus of algebraic curves of degree $q$, given by the Plücker formula. Recall that for a mixed strongly polar homogeneous polynomial, the genus and the Euler characteristic of the Milnor fiber are related as follows ( by (2) of Proposition 3):

\section{Proposition 14.}

$$
2-2 g=3-\frac{\chi(F)}{q}
$$

where

$$
F=\left\{\left(z_{1}, z_{2}, z_{3}\right) \in \mathbb{C}^{3} \mid f\left(z_{1}, z_{2}, z_{3}, \bar{z}_{1}, \bar{z}_{2}, \bar{z}_{3}\right)=1\right\}
$$


Now we will see some examples which shows that $\chi(F)$ is not an invariant of $q$.

I. Simplicial polynomials. We consider the following simplicial polar homogeneous polynomials of polar degree $q$.

$$
\begin{aligned}
& f_{s_{1}}(\mathbf{z}, \overline{\mathbf{z}})=z_{1}^{q+r} \bar{z}_{1}^{r}+z_{2}^{q+r} \bar{z}_{2}^{r}+z_{3}^{q+r} \bar{z}_{3}^{r} \\
& f_{s_{2}}(\mathbf{z}, \overline{\mathbf{z}})=z_{1}^{q+r-1} \bar{z}_{1}^{r} z_{2}+z_{2}^{q+r-1} \bar{z}_{2}^{r} z_{3}+z_{3}^{q+r} \bar{z}_{3}^{r} \\
& f_{s_{3}}(\mathbf{z}, \overline{\mathbf{z}})=z_{1}^{q+r-1} \bar{z}_{1}^{r} z_{2}+z_{2}^{q+r-1} \bar{z}_{2}^{r} z_{3}+z_{3}^{q+r-1} \bar{z}_{3}^{r} z_{1} \\
& f_{s_{4}}(\mathbf{z}, \overline{\mathbf{z}})=z_{1}^{q+r+1} \bar{z}_{1}^{r} \bar{z}_{2}+z_{2}^{q+r+1} \bar{z}_{2}^{r} \bar{z}_{3}+z_{3}^{q+r} \bar{z}_{3}^{r} \\
& f_{s_{5}}(\mathbf{z}, \overline{\mathbf{z}})=z_{1}^{q+r+1} \bar{z}_{1}^{r} \bar{z}_{2}+z_{2}^{q+r+1} \bar{z}_{2}^{r} \bar{z}_{3}+z_{3}^{q+r+1} \bar{z}_{3}^{r} \bar{z}_{1}
\end{aligned}
$$

Let $F_{s_{i}}$ be the Milnor fiber of $f_{s_{i}}$ and let $C_{s_{i}}$ be the corresponding projective curves for $i=1, \ldots, 5$. First, the Euler characteristic of the Milnor fibers and the genera are given by Proposition 13 and Proposition 14 as follows.

$$
\begin{aligned}
& \chi\left(F_{s_{i}}\right)=q^{3}-3 q^{2}+3 q, \quad g\left(C_{s_{i}}\right)=\frac{(q-1)(q-2)}{2}, i=1,2,3 \\
& \chi\left(F_{s_{4}}\right)=q\left(q^{2}+q+1\right), \quad g\left(C_{s_{4}}\right)=\frac{q(q+1)}{2} \\
& \chi\left(F_{s_{5}}\right)=q\left(q^{2}+3 q+3\right), \quad g\left(C_{s_{4}}\right)=\frac{(q+2)(q+1)}{2}
\end{aligned}
$$

In [9], we have shown that $C_{s_{1}}$ and $C_{s_{2}}$ are isomorphic to algebraic plane curves defined by the associated homogeneous polynomials of degree $q$ :

$$
\begin{aligned}
& g_{s_{1}}(\mathbf{z})=z_{1}^{q-1} z_{2}+z_{2}^{q-1} z_{3}+z_{3}^{q} \\
& g_{s_{2}}(\mathbf{z})=z_{1}^{q-1} z_{2}+z_{2}^{q-1} z_{3}+z_{3}^{q} .
\end{aligned}
$$

We also expect that $C_{s_{3}}$ is isotopic to the algebraic curve

$$
z_{1}^{q-1} z_{2}+z_{2}^{q-1} z_{3}+z_{3}^{q-1} z_{1}=0
$$

as the genus of $C_{s_{3}}$ suggests it (see also [9]).

II. We consider the following join type polar homogeneous polynomial.

$$
\begin{aligned}
& h_{j}(\mathbf{z}, \overline{\mathbf{z}})= g_{j}(\mathbf{w}, \overline{\mathbf{w}})+z_{3}^{q+r} \bar{z}_{3}^{r}, \\
& g_{j}(\mathbf{w}, \overline{\mathbf{w}})=\left(w_{1}^{q+j} \bar{w}_{1}^{j}+w_{2}^{q+j} \bar{w}_{2}^{j}\right)\left(w_{1}^{r-j}-\alpha w_{2}^{r-j}\right)\left(\bar{w}_{1}^{r-j}-\beta \bar{w}_{2}^{r-j}\right), \\
& 0 \leq j \leq r .
\end{aligned}
$$

$\left(\alpha, \beta \in \mathbb{C}^{*}\right.$ are generic.) The the Milnor fiber $F_{g_{j}}$ of $g_{j}$ is connected. The Euler characteristic of $\chi\left(F_{g_{j}}^{*}\right)\left(F_{g_{j}}^{*}=F_{g_{j}} \cap \mathbb{C}^{* 2}\right)$ is given by $\chi\left(F_{g_{j}}^{*}\right)=-r_{g} q$ where $r_{g}$ is the link component number of $g=0$ which is $q+2(r-j)$. Thus $\chi\left(F_{g_{j}}\right)=\chi\left(F_{g_{j}}^{*}\right)+2 q$ where the last terms come from $\left.\chi\left(F_{g_{j}}\right)^{* I}\right)$ with $I=\{1\}$ or $\{2\}$. Thus $\chi\left(F_{g_{j}}\right)=q(q-2+2(r-j))$ and $g=\frac{(q-1)(q-2+2(r-j))}{2}$. We observe that the genus can take the following values by taking $j=r, \ldots, 0$ :

$$
\frac{(q-1)(q-2)}{2}, \frac{(q-1) q}{2}, \ldots, \frac{(q-1)(q+2 r-2)}{2} .
$$


As we can take the positive number $r$ arbitrary large, we have that

Proposition 15. There exist differentiable curves embedded in $\mathbb{C P}^{2}$ with a fixed degree $q \geq 2$ whose genera are given as:

$$
\left\{g_{0}+k(q-1) \mid k=0,1, \ldots\right\}, \quad g_{0}:=\frac{(q-1)(q-2)}{2} .
$$

In particular, taking $q=2$, we obtain:

Corollary 16. For any smooth surface $S$ of genus $g$, there is an embedding $S \subset \mathbb{C P}^{2}$ so that the degree of $S$ is 2 .

\section{REFERENCES}

[1] A. Borel and A. Haefliger. La classe d'homologie fondamentale d'un espace analytique. Bull. Soc. Math. France, 89:461-513, 1961.

[2] J. L. Cisneros-Molina. Join theorem for polar weighted homogeneous singularities. In Singularities II, volume 475 of Contemp. Math., pages 43-59. Amer. Math. Soc., Providence, RI, 2008.

[3] P. Griffiths and J. Harris. Principles of algebraic geometry. Wiley Classics Library. John Wiley \& Sons Inc., New York, 1994. Reprint of the 1978 original.

[4] P. B. Kronheimer and T. S. Mrowka. The genus of embedded surfaces in the projective plane. Math. Res. Lett., 1(6):797-808, 1994.

[5] J. Milnor. Singular points of complex hypersurfaces. Annals of Mathematics Studies, No. 61. Princeton University Press, Princeton, N.J., 1968.

[6] M. Oka. On the cohomology structure of projective varieties. In Manifolds-Tokyo 1973 (Proc. Internat. Conf., Tokyo, 1973), pages 137-143. Univ. Tokyo Press, Tokyo, 1975.

[7] M. Oka. Topology of polar weighted homogeneous hypersurafces. Kodai Math. J., 31(2):163-182, 2008.

[8] M. Oka. Non-degenerate mixed functions. ArXiv 0909.1904, to appear in Kodai Math. J., 2009.

[9] M. Oka. On mixed Brieskorn variety. ArXiv 0909.4605v2, 2009.

[10] E. H. Spanier. Algebraic topology. McGraw-Hill Book Co., New York, 1966.

Department of MAThematics

Tokyo University of Science

26 WAKAmiYa-CHO, ShinJuKu-KU

TOKYO 162-8601

E-mail: oka@rs.kagu.tus.ac.jp 